\title{
Dénombrement des germes totaux et des bactéries coliformes des laits crus délivrés à la Centrale laitière de Téhéran, et détermination du taux de contamination de ces laits en staphylocoques coagulase-positive
}

\author{
par \\ R. GHAZVINIAN, A. FARKHONDEH, M. MALEKI \\ et M. KOHNECHAHRI \\ Département d'Hygiène Alimentaire de l'Université de Téhéran
}

\section{Introduction}

Dans un autre travail, Ghazvinian et Maleki [1] ont déterminé le taux de contamination du rhino-pharynx et de la main chez le personnel des différentes usines alimentaires de la région téhéranaise en staphylocoques coagulase-positive. Sur 745 prélèvements, le pourcentage de contamination en staphylocoques coagulase-positive était de 35,5 p. 100 pour la fosse nasale, 33 p. 100 pour la main et 9,4 p. 100 pour la gorge.

L'importance des intoxications alimentaires attribuées aux staphylocoques coagulase-positive dans la ville de Téhéran, d'une population de plus de 3000000 hab., nous a amené à faire une étude sur la contamination par ces germes de quelques aliments d'origine animale avant le traitement thermique.

$\mathrm{Au}$ cours de ce travail, nous nous sommes proposés de dénombrer les germes totaux et les bactéries coliformes des laits de livraison à la Centrale laitière gouvernementale de Téhéran en déterminant le taux de contamination de ces laits en staphylocoques coagulase-positive.

\section{Protocole expérimental}

Au cours d'une période allant du 15 mars 1970 à la fin de février 1971, nous avons prélevé 1000 échantillons de lait de 120 éleveurs produisant du lait pour la Centrale laitière de Téhéran. A l'exception d'une seule ferme, dont le lait est transporté en camion-citerne, 
TABLEAU 1

Température, Pluviométrie et Humidité relatives pour les mois de l'année dans la région de Téhéran (période de 1943-1965) (Latitude : 35 $41^{\prime} \mathrm{N}$ - Longitude : 51 $19^{\prime} \mathrm{E}$ - Hauteur : $1198 \mathrm{~m}$ )

\begin{tabular}{|c|c|c|c|c|c|c|c|c|c|c|c|c|c|c|}
\hline \multirow[b]{2}{*}{ Mois de l'année } & \multicolumn{5}{|c|}{ Température $\left({ }^{\circ} \mathrm{C}\right)$} & \multicolumn{4}{|c|}{ Pluviométrie (mm) } & \multicolumn{2}{|c|}{$\begin{array}{c}\text { Humidité } \\
\text { relative }\end{array}$} & \multicolumn{2}{|c|}{$\begin{array}{l}\text { Nombre de } \\
\text { jours }\end{array}$} & \multirow[b]{2}{*}{$\frac{\overrightarrow{0}}{0}$} \\
\hline & 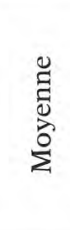 & 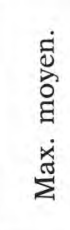 & 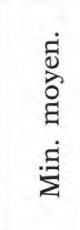 & 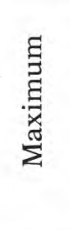 & 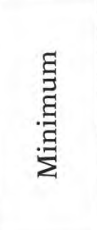 & 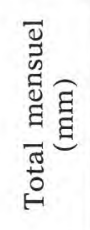 & 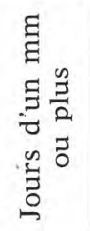 & 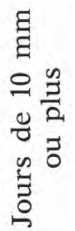 & 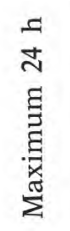 & $\begin{array}{l}\sum_{0}^{E} \\
0\end{array}$ & $\begin{array}{l}\sum_{0}^{E} \\
8\end{array}$ & $\vec{v}$ & $\begin{array}{l}\stackrel{2}{0} \\
\ddot{z}\end{array}$ & \\
\hline Janvier & 3,2 & 8,1 & $-1,7$ & 18,6 & $-16,1$ & 38,2 & 5,3 & 1,0 & 25,1 & 78 & 55 & 19 & 3 & 186,0 \\
\hline Février & 5,5 & 10,8 & 0,2 & 23,0 & $-12,2$ & 27,0 & 4,4 & 1,0 & 39,7 & 66 & 41 & 14 & 1,5 & 185,0 \\
\hline Mars & 9,8 & 15,5 & 4,1 & 26,1 & $-5,6$ & 32,3 & 4,9 & 0,5 & 12,3 & 61 & 35 & 5 & 0,3 & 222,0 \\
\hline Avril & 15,3 & 21,3 & 9,3 & 32,0 & $-4,1$ & 25,4 & 6,1 & 1,2 & 39,0 & 57 & 30 & 0,2 & - & 310,0 \\
\hline Mai & 21,6 & 28,1 & 15,1 & 36,2 & 2,0 & 13,6 & 3,2 & 0,2 & 9,0 & 42 & 21 & - & - & 209,0 \\
\hline Juin & 26,4 & 33,2 & 19,6 & 40,1 & 8,9 & 2,0 & 1,0 & - & 3,1 & 34 & 13 & - & - & 349,0 \\
\hline Juillet : & 29,5 & 36,4 & 22,6 & 42,5 & 14,7 & 0,6 & 0,3 & - & 0,6 & 32 & 17 & - & - & 356,0 \\
\hline Août & 28,5 & 35,2 & 21,8 & 42,2 & 11,1 & 1,7 & 0,1 & $\overline{0,1}$ & $\begin{array}{r}0,0 \\
12,0\end{array}$ & 32 & 18 & - & - & 340,0 \\
\hline Septembre & 24,9 & 31,2 & $\begin{array}{l}21,0 \\
18,6\end{array}$ & $\begin{array}{l}42,2 \\
38,0\end{array}$ & $\begin{array}{r}11,1 \\
8,3\end{array}$ & $\begin{array}{l}1,1 \\
1,5\end{array}$ & 0,3 & - & 2,2 & 32 & 18 & - & - & 309,4 \\
\hline Octobre & 18,3 & 24,6 & $\begin{array}{l}10,0 \\
12,0\end{array}$ & 34,4 & 1,7 & 5,6 & 1,5 & $\overline{0,1}$ & $\begin{array}{r}2,2 \\
15,3\end{array}$ & 42 & 24 & - & - & 270,0 \\
\hline Novembre & 10,7 & 16,1 & 5,3 & 25,0 & $-7,5$ & 27,0 & 3,5 & 1,0 & 19,5 & 59 & 35 & 2 & 0,1 & 209,0 \\
\hline Décembre & 5,1 & 9,0 & 0,5 & 19,4 & $-15,0$ & 27,4 & 4,6 & 0,5 & 38,8 & 74 & 48 & 10 & 1,5 & 191,0 \\
\hline Année & 16,5 & 22,5 & 10,6 & 42,5 & $-16,1$ & 202,3 & 35,2 & 5,6 & 39,7 & 51 & 30 & 50,2 & 6,4 & 3136,0 \\
\hline
\end{tabular}


$T A B L E A U 2$

Taux de contamination des laits crus délivrés à la Centrale laitière de Téhéran en staphylocoques coagulase-positive

\begin{tabular}{|c|c|c|c|c|c|c|}
\hline Saison & $\begin{array}{c}\text { Nombre } \\
\text { d'échantillons }\end{array}$ & Positifs & $\begin{array}{c}\text { p. } 100 \text { des cas } \\
\text { positifs }\end{array}$ & Max. & Min. & Moyenne \\
\hline Printemps & 294 & 48 & 16,3 & $7 \times 10^{4}$ & $3 \times 10^{1}$ & $5 \times 10^{3}$ \\
\hline Eté & 169 & 12 & 7,1 & $2 \times 10^{5}$ & $1 \times 10^{2}$ & $2 \times 10^{4}$ \\
\hline Automne & 244 & 29 & 11,8 & $4 \times 10^{3}$ & $1 \times 10^{2}$ & $5 \times 10^{2}$ \\
\hline Hiver & 293 & 54 & 18,4 & $2 \times 10^{4}$ & $1 \times 10^{2}$ & $1 \times 10^{3}$ \\
\hline Toute l'année & 1000 & 143 & 13,4 & $2 \times 10^{5}$ & $3 \times 10^{1}$ & $6 \times 10^{3}$ \\
\hline
\end{tabular}


tout le lait est arrivé au quai de réception en bidons de 20 à 501 . Sur 120 fermes, 45 seulement possédaient des tanks de réfrigération du lait. Pour chaque ferme, des prélèvements ont été effectués deux fois par saison. Le rayon de ramassage du lait pour la Centrale laitière de Téhéran, traitant à l'heure actuelle 1800001 de lait/j, est au maximum de l'ordre de $60 \mathrm{~km}$. Le tableau 1 montre la température, la pluviométrie et l'humidité relative pour les différents mois de l'année de la région de Téhéran.

\section{Méthode et matériel}

Les prélèvements ont été effectués conformément aux normes internationales FIL [2, 3]. Un échantillon est prélevé sur chacun des bidons pris au hasard en utilisant un plongeur, en acier inoxydable, préalablement stérilisé à l'eau bouillante, et après avoir mélangé soigneusement le lait à l'aide d'un agitateur stérilisé de même façon. Les flacons stériles contenant les échantillons sont gardés dans la glace et transportés le plus rapidement possible vers le laboratoire.

Chaque échantillon est utilisé pour l'ensemencement en vue de la numération totale, dénombrement des bactéries coliformes et des staphylocoques coagulase-positive. Les milieux suivants ont été utilisés :

- Milieu de référence [4] pour la numération totale.

- Violet Red Bile Agar [5] pour le dénombrement des bactéries coliformes. coques.

- Milieu Baird-Parker $[6,11]$ pour la recherche des staphylo-

La température et la durée d'incubation utilisées étaient pour la numération des germes totaux de $30^{\circ} \mathrm{C} / 72 \mathrm{~h}$, pour le dénombrement des bactéries coliformes de $30^{\circ} \mathrm{C} / 24 \mathrm{~h}$ et pour la recherche des staphylocoques de $37^{\circ} \mathrm{C} / 48 \mathrm{~h}$.

Les colonies de staphylocoques ont été isolées et ensemencées sur le milieu Trypticase soya agar BBL en vue de la recherche ultérieure de la coagulase et dans le cas où le test de la coagulase était positif, le typage de bactériophage était effectué.

\section{Résultats}

Le tableau 2 montre le taux de contamination des laits crus de ferme en staphylocoques coagulase-positive.

Sur 1000 échantillons examinés, le nombre des cas positifs est de $143(13,4 \mathrm{p}, 100)$ avec un minimum de $3 \times 10^{1}$, un maximum de $2 \times 10^{5}$ et une moyenne de $6 \times 10^{3}$ au cours de l'année. Les tableaux 1 et 2 nous montrent que pour une température plus élevée le nombre des cas positifs de contamination en staphylocoques coagulasepositive est plus bas. 
TABLEAU 3

Résultats de la numération des germes totaux et des bactéries coliformes des laits crus délivrés à la Centrale laitière de Téhéran

\begin{tabular}{|c|c|c|c|c|c|c|c|}
\hline \multirow{2}{*}{ Saison } & \multirow{2}{*}{$\begin{array}{c}\text { Nombre } \\
\text { d'échantillons }\end{array}$} & \multicolumn{3}{|c|}{ Dénombrement des germes totaux } & \multicolumn{3}{|c|}{ Bactéries coliformes } \\
\hline & & Max. & Min. & Moyenne & Max. & Min. & Moyenne \\
\hline Printemps & 294 & $8 \times 10^{\mathrm{s}}$ & $2 \times 10^{6}$ & $15 \times 10^{7}$ & $3 \times 10^{6}$ & $1 \times 10^{2}$ & $1 \times 10^{8}$ \\
\hline Eté & 169 & $5 \times 10^{8}$ & $2 \times 10^{8}$ & $8 \times 10^{7}$ & $7 \times 10^{8}$ & $9 \times 10^{1}$ & $1 \times 10^{8}$ \\
\hline Automne & 244 & $41 \times 10^{7}$ & $13 \times 10^{4}$ & $3 \times 10^{7}$ & $7 \times 10^{8}$ & $1 \times 10^{2}$ & $4 \times 10^{5}$ \\
\hline Hiver & 293 & $19 \times 10^{7}$ & $7 \times 10^{4}$ & $2 \times 10^{7}$ & $8 \times 10^{8}$ & $1 \times 10^{2}$ & $4 \times 10^{5}$ \\
\hline Toute l'année & 1000 & $8 \times 10^{8}$ & $7 \times 10^{4}$ & $7 \times 10^{7}$ & $8 \times 10^{6}$ & $9 \times 10^{1}$ & $8 \times 10^{5}$ \\
\hline
\end{tabular}


Le tableau 3 montre les résultats de la numération des germes totaux et le dénombrement des bactéries coliformes des laits crus délivrés à la Centrale laitière de Téhéran.

D'après ce tableau, le nombre maximum pour les germes totaux est de $8 \times 10^{s}$, le minimum $7 \times 10^{4}$ et la moyenne $7 \times 10^{7}$, et, pour les bactéries coliformes, de $8 \times 10^{6}, 9 \times 10^{1}$ et $8 \times 10^{5}$.

Le tableau 4 montre les résultats du dénombrement des germes totaux, des bactéries coliformes et des staphylocoques coagulasepositive des laits crus des fermes ayant des appareils de réfrigération du lait.

On voit par ce tableau que le nombre des germes totaux et des bactéries coliformes est plus bas dans le lait provenant de ces fermes tandis que le pourcentage de la contamination en staphylocoques coagulase-positive est relativement plus élevé.

Les tableaux 5 et 6 montrent les types de bactériophages des staphylocoques isolés des laits crus de livraison à la Centrale laitière de Téhéran avec le pourcentage correspondant.

D'après le tableau 6, 18,1 p. 100 des staphylocoques coagulasepositive ont été de types mixtes et dans un seul cas le phage $42 \mathrm{D}$ a été lysé et, comme il était accompagné d'autres phages, nous l'avons groupé en types mixtes.

\section{Commentaires}

Le taux de contamination du lait cru en staphylocoques coagulasepositive est variable selon les conditions de production. Mc Philipps et Cuningham [7] ont trouvé le taux maximum de $10^{4} / \mathrm{ml}$ de lait cru de tank de qualité A. D'après Murray [8] 30 p. 100 des laits crus étaient exempts de staphylocoques et $1,6 \mathrm{p} .100$ seulement contenaient plus de $10^{3} / \mathrm{ml}$. Seelman, Oliger, Rachov et Welz [9] n'ont pas trouvé de staphylocoques dans 68 p. 100 des laits crus et 11 p. 100 seulement des échantillons contenaient moins de $10^{3} / \mathrm{ml}$. Sharpe, Fewins, Reitz et Cuthbert [10] ont montré que 5 p. 100 seulement des échantillons contenaient moins de $10^{2} / \mathrm{ml}$ et $30 \mathrm{p} .100$ plus de $10^{4}$.

Dans notre expérience sur le lait de 120 producteurs, dans une période de quatre saisons, nous n'avons pas rencontré de staphylocoques coagulase-positive dans 86,6 p. 100 des cas. Mais dans 45 fermes ayant des appareils de réfrigération du lait, le pourcentage des cas négatifs était de 77 p. 100. On voit d'ailleurs bien qu'avec une température plus élevée et en l'absence de réfrigérants le nombre des germes totaux et des bactéries coliformes est plus élevé tandis que le nombre des staphylocoques est plus bas. Cela prouve l'influence certaine de la croissance des autres germes et en particulier, les ferments lactiques sur le développement des staphylocoques dans les laits produits et collectés dans de mauvaises conditions. 
TABLEAU 4

Résultats de dénombrement des germes totaux, des bactéries coliformes et des staphylocoques coagulase-positive des laits de fermes ayant des appareils de réfrigération du lait

\begin{tabular}{|c|c|c|c|c|c|c|c|c|c|c|c|}
\hline \multirow[b]{2}{*}{$\begin{array}{c}\text { Nombre } \\
\text { d'échantillons }\end{array}$} & \multicolumn{5}{|c|}{ Staphylocoques coagulase +} & \multicolumn{3}{|c|}{ Germes totaux } & \multicolumn{3}{|c|}{ Bactéries coliformes } \\
\hline & $\begin{array}{c}\text { Cas } \\
\text { positifs }\end{array}$ & $\begin{array}{l}\text { p. } 100 \text { des } \\
\text { cas } \\
\text { positifs }\end{array}$ & Max. & Min. & Moyenne & Max. & Min. & Moyenne & Max. & Min. & Moyenne \\
\hline 303 & 70 & 23,1 & 80,000 & 100 & 1750 & $15 \times 10^{7}$ & $7 \times 10^{4}$ & $19 \times 10^{6}$ & $3 \times 10^{6}$ & $1 \times 10^{3}$ & $3 \times 10^{5}$ \\
\hline
\end{tabular}

TABLEAU 5

Types de bactériophages des staphylocoques isolés des laits crus délivrés à la Centrale laitière de Téhéran

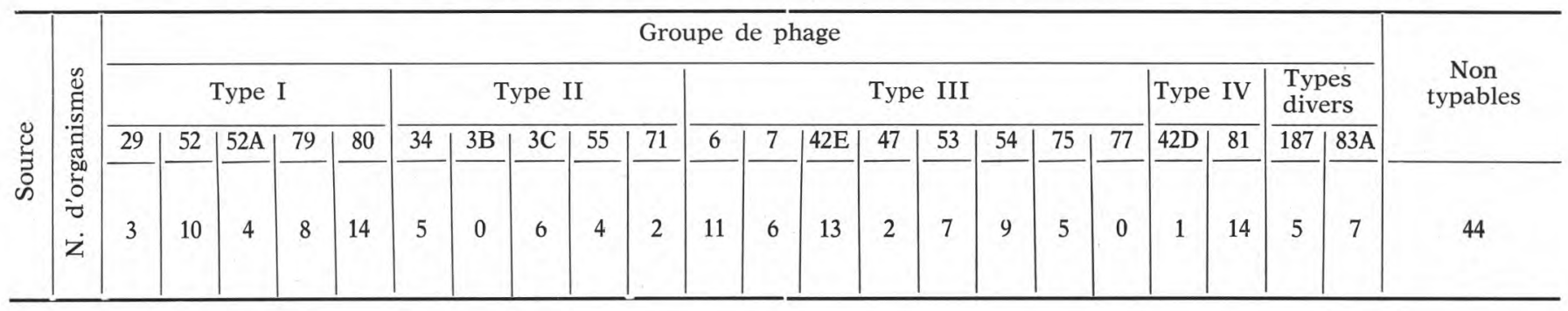

TABLEAU 6

Pourcentage des staphylocoques isolés sensibles aux bactériophages

\begin{tabular}{|c|c|c|c|c|c|c|c|c|}
\hline $\begin{array}{c}\text { Source } \\
\text { de contamination }\end{array}$ & $\begin{array}{c}\text { Nombre } \\
\text { d'examens }\end{array}$ & I & II & III & IV & Mixtes & Divers & $\begin{array}{c}\text { Non } \\
\text { typabies }\end{array}$ \\
\hline Lait cru & 93 & 7,5 & 5,3 & 11,8 & - & 18,1 & 9,6 & 47,3 \\
\hline
\end{tabular}




\section{S u m m a ry}

1000 samples of raw milk from 120 farms producing milk for the Tehran pasteurizing Plant have been studied for total and coli count with special reference to coagulase-positive staphylococci. According to the results obtained, in 86,6 p. 100 of the cases no coagulase-positive staphylococci has been found but in 45 samples coming from the farms equipped with cooling apparatus the percentage of positive cases is $23,1 \mathrm{p} .100$. This is certainely due to the influence of the growth of other microorganisms and in particular the lactic acid bacteria on the staphylococcal strains.

\section{Remerciements}

Nous tenons à remercier $M$. le Pr $H$. Neshat, Directeur du Département d'hygiène alimentaire de l'Université de Téhéran, pour les conseils qu'il a bien voulu nous adresser au cours de ce travail. Nous remercions également les responsables de la Centrale laitière de Téhéran pour les facilités qu'ils nous ont faites et enfin nous remercions nos collègues du Centre de typage des bactériophages de la Faculté d'Hygiène de l'Université de Téhéran pour l'aide qu'ils nous ont apportée pour la classification des bactériophages.

Reçu pour publication en août 1971.

\section{Bibliographie}

[1] Ghazvinian (R) and Maleki (M.) (1971). - Nasal, Hand and Throat Carriage of Coagulase-positive Staphylococcus aureus. In Food Handlers in Tehran (sous presse).

[2] Methods of sampling milk and milk products. International Standard FIL/ IDF, 2, 1958.

[3] Standard methods for sampling milk and milk products. International Standard FIL/IDF, 50, 1969.

[4] Colony count of liquid milk and dried milk (1958). International Standard FIL/IDF, 3, 1958.

[5] Méthode de routine normalisée pour le dénombrement des bactéries coliformes dans le lait cru. Norme Internationale FIL/IDF, 39, 1966.

[6] Baird Parcker (A. C.) (1962). - J. Appl. Bact., 25, 12.

[7] Mc Philtiss (J.), and Cunningham (E. A.) (1962). - Aus. J. Dairy Technol., $17,95$.

[8] Murray (J. G.) (1963). - J. Soc. Dairy Technol., 15, 25.

[9] Seelman (M.), Obiger (G.), Rachov (H. G.) and Wrlz (W.) (1963). - Dairy Sci. Abstr., 25, 3270.

[10] Sharpe (M. E.), Neave (F. K.) and Reiter (B.) (1962). - J. Appl. Bact., $25,403$.

[11] Thieulin (G.), Basille (D.), Pantaléon (J.), Rosset (R.), Gandon (Y.) et Petit (A.) (1966). - Recherche des staphylocoques pathogènes dans le lait et les produits laitiers. Le Lait, $\mathrm{n}^{\circ} 453-454,131$. 